\title{
Simulation of Augmented Reality Systems in Purely Virtual Environments
}

\author{
Eric Ragan, Curtis Wilkes, and Doug A. Bowman ${ }^{1}$ \\ Department of Computer Science \\ Virginia Tech \\ Blacksburg, VA 24061
}

\author{
Tobias Höllerer ${ }^{2}$ \\ Department of Computer Science \\ University of California at Santa Barbara \\ Santa Barbara, CA 93106
}

\begin{abstract}
We propose the use of virtual environments to simulate augmented reality (AR) systems for the purposes of experimentation and usability evaluation. This method allows complete control in the AR environment, providing many advantages over testing with true AR systems. We also discuss some of the limitations to the simulation approach. We have demonstrated the use of such a simulation in a proof of concept experiment controlling the levels of registration error in the $A R$ scenario. In this experiment, we used the simulation method to investigate the effects of registration error on task performance for a generic task involving precise motor control for AR object manipulation. Isolating jitter and latency errors, we provide empirical evidence of the relationship between accurate registration and task performance.
\end{abstract}

KEYwORDS: Augmented reality, AR simulation, registration error

\section{AR SIMULATION}

Due to far greater control over both the virtual and simulated real components, the concept of using virtual reality (VR) to simulate an augmented reality system has many advantages over testing approaches that rely on AR systems. Though researchers and designers should be mindful of the method's limitations, it has the potential to facilitate promising advancements in the realm of AR.

\subsection{Benefits of AR Simulation}

The concept of using VR to simulate a complete AR system has several advantages over an actual AR environment. For instance, such an arrangement makes it possible to precisely control the registration of virtual objects, allowing testing of exact levels of registration error. Such an approach even enables the ability to test results of "perfect" registration, which is impossible when using real AR systems (though VR systems also suffer from registration error-see the next section for discussion). The complete registration control also makes it possible to isolate and independently manipulate different types of registration error, allowing studies of interactions among the types of error, which actual AR technology does not allow. Simulation can also facilitate the manipulation of other factors of the augmented display, such as field of view or image resolution.

As a further general benefit, simulated AR allows researchers to test systems or hardware that do not actually exist. The

\footnotetext{
${ }^{1}$ \{eragan, cbwilkes, dbowman $\} @$ vt.edu

2 holl@cs.ucsb.edu
}

effectiveness of system designs can be tested without expensive implementations or additional devices. Additional benefit may be found in virtual reality's ability to precisely control the simulated real environment. This could allow AR implementations to be easily tested and optimized before deployment in real-life locations and situations that are not easily available for a complete usability engineering life cycle. Another advantage is complete control over what happens in the simulated real environment makes it possible to test a system in a wide variety of use scenarios, including those that might be too difficult, dangerous, or costly to produce in the real world (e.g., AR support for firefighters). These capabilities can certainly be useful throughout the design and evaluation processes.

\subsection{Challenges in AR Simulation}

AR simulation also has clear challenges related to the fidelity of the real world component in the system. Though some issues can be managed through design considerations, others may be more problematic. One issue, for example, is the lack of tactile feedback in the simulated real environment. This may not be problematic, however, if the simulation does not require or allow interactions with the simulated physical objects.

Another disadvantage is that the simulation approach does not allow users to physically walk large distances due to size limitations of VR platforms. This issue may require additional consideration if the test system simulates a physically large area and virtual travel techniques might interfere with the investigation.

Another issue is the tracking error within the virtual environment itself, which will mean that the registration of the simulated real environment cannot be perfect. In modern VR systems, however, the perceived error will be low, and may even be unnoticeable. Although the trackers in any VR system will introduce some degree of latency and jitter, such error usually has low impact because all virtual objects are affected equally [e.g. 3]. By contrast, in AR, only the virtual objects exhibit error, resulting in a mismatch between the real and virtual parts of the scene.

Additionally, virtual reality implementations suffer from limitations in luminance. Because VR systems cannot produce very bright displays, they may not be able to replicate the brightness of some outdoor scenarios. With these systems, it may be difficult to investigate the effects of properties such as the relative brightness and contrast between real and virtual objects or the level of opacity of the virtual objects.

VR also presents different depth cues than those experienced in the real world of AR. Even though stereoscopic imagery can offer convergence cues, the current methods used to display virtual objects cannot enable the use of ocular accommodation cues because the objects are always in focus at the depth of the projection screen. Because all objects in VR are virtual, they all provide the same imperfect visual depth cues. In an $A R$ 
environment, on the other hand, while the virtual components suffer from the same types of imperfect cues, the real world objects will provide perfect depth cues for optical see-through AR systems. As a result, the distinction between real and virtual objects in a simulated AR environment will differ from the corresponding disparity in an actual AR system.

Though an AR simulation does not provide a perfect representation of an actual AR system, the simulation approach still has the potential to provide great benefit to AR research. Additionally, as technological advancements further the realism of virtual reality systems and reduce these limitations, the quality of the simulations will also improve.

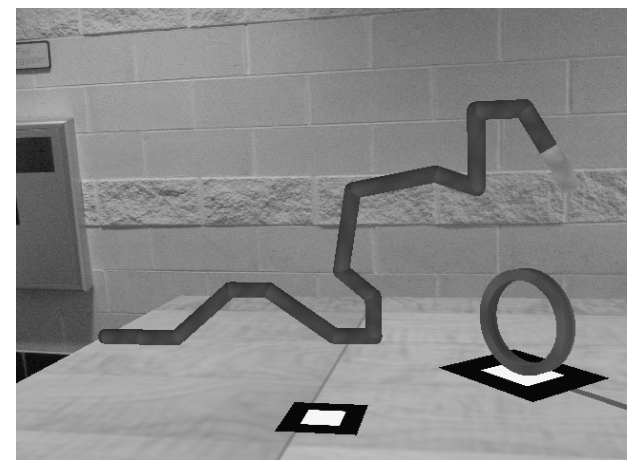

Figure 1. Virtual tube path and ring augmented over simulated real marker squares in the simulated real room

\section{Proof of Concept Experiment}

We sought to provide proof of concept showing that VR can be used to simulate an AR system in a meaningful way. In this study, we explored challenges inherent in such a simulation and aimed to determine whether such a setup can be used productively for AR research. We used a simulated AR system to investigate the effects of registration error on performance for tasks requiring accurate manipulation of augmented objects. The scenario was simulated using a four-sided CAVE with an Intersense IS-900 tracking system for head and hand tracking.

We sought confirmation of the relationship between latency and performance shown by Ellis in an actual AR system [1]. Similar to the tasks of Ellis' study, our task required a user to guide a ring along a crooked path shown by a bending tube structure (Figure 1). In our setup, the virtual tube path was augmented over the rendition of an ARToolKit marker on a table in our simulated real world environment. The virtual ring was augmented over another marker that was attached to the end of a stick. The main goal of the task was to move the ring from one end of the tube to the other while keeping the tube inside the ring and limiting collisions.

The types of registration error controlled in the simulation were jitter and latency. As static tracker error, jitter is output noise exhibited as translation and orientation offset. Latency is the result of all system delays from the time scene motion occurs up to the time of image generation and display [2]. For the jitter conditions, levels of error were controlled through specification of the standard deviation of the normal distribution. The translational standard deviations used were $0,3.81,7.62$, and $11.43 \mathrm{~mm}$. Based on the positional standard deviation, an angular offset was also determined for each degree of freedom (yaw, pitch, and roll). We simulated latency errors of $0,176,352$, and $528 \mathrm{~ms}$ by controlling frame delays for the simulated virtual ring.

The total time taken to complete each trial and the percentage of time colliding were measured as performance metrics.
Statistical analysis of both metrics showed significant main effects for both jitter and latency. Further analysis also showed an interaction between the latency and jitter conditions for completion time (Figure 2). This interaction is related to the fact that users took less time in the presence of jitter. With no jitter, completion time follows a linear increase as latency increases. In the presence of jitter, however, the rate of increase in completion time is lower for the same increases in latency. This interaction between jitter and latency is a novel result, and was only found because the use of an AR simulator made it possible to independently control these two components of AR immersion. These results suggest that jitter was the dominant type of error. These findings encourage further pursuit of the concept of using VR systems to simulate AR and conduct usability research.

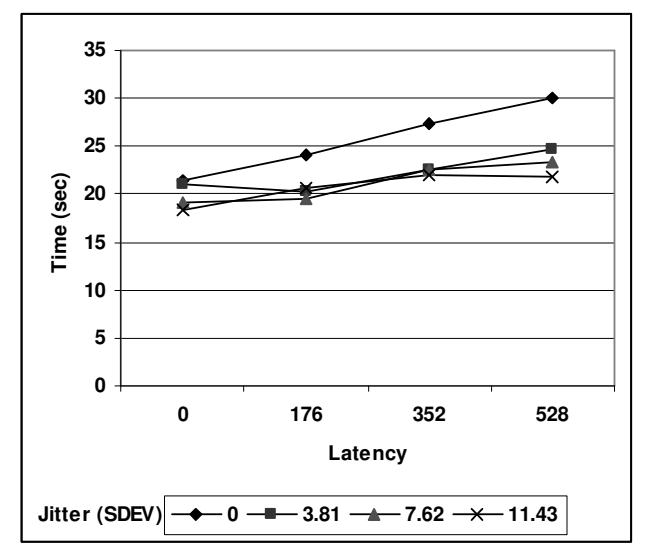

Figure 2. Interaction between latency and jitter

\section{ConcLusion}

We propose the use of VR systems to simulate AR scenarios to help overcome some of the difficulties in evaluating usability in AR. In addition to explaining several benefits to the simulation approach, we have demonstrated that levels of registration error can be controlled through such a setup through a proof of concept experiment. Future studies using AR simulators could take advantage of VR systems with higher levels of immersion (e.g., fully-surrounding displays, higher resolution, greater brightness levels) to simulate more realistic models of the real world scene. While we focused on registration error in our study, AR simulators should be able to control many more components of AR immersion, such as the real and virtual fields of view, relative brightness and contrast between real and virtual components, level of opacity of the virtual objects, or resolution of virtual objects. Further work is needed to test the validity of using VR to research $\mathrm{AR}$ in order to reveal the true potential or expose additional weaknesses to this approach.

\section{References}

[1] Ellis, S.R. et al. 1997. Factors influencing operator interaction with virtual objects viewed via head-mounted see-through displays: viewing conditions and rendering latency. Proceedings of Virtual Reality Annual International Symposium 1997. IEEE CS Press. Los Alamitos, California, 1997. Pages 138-145.

[2] Holloway, R. 1997. Registration Error Analysis for Augmented Reality. Presence: Teleoperators and Virtual Environments, 1997.

[3] Sprague, D. Po, B. Booth, K. 2006. The Importance of Accurate VR Head Registration on Skilled Motor Performance. ACM International Conference Proceeding Series; Vol. 137 Proceedings of Graphics Interface 2006. Pages 131-137. 